\title{
Polymorphisms in Gene UGT1A1 Modify the Association of Prenatal Exposure to Polycyclic Aromatic Hydrocarbons With Congenital Heart Diseases Risk
}

\section{Jing Tao}

National Office for Maternal and Child Health Surveillance of China, Sichuan University

Nana Li

National Office for Maternal and Child Health Surveillance of China, Sichuan University

Zhen Liu

National Office for Maternal and Child Health Surveillance of China, Sichuan University

\section{Ying Deng}

National Office for Maternal and Child Health Surveillance of China, Sichuan University

Xiaohong Li

National Office for Maternal and Child Health Surveillance of China, Sichuan University

Fangfang Luo

Meishan maternal and child health care hospital, Sichuan, China.

Yanna Zou

Changyi maternal and child health care hospital

\section{Ping Yu}

National Office for Maternal and Child Health Surveillance of China, Sichuan University

Jun Zhu ( $\nabla$ zhujun028@163.com )

National Office for Maternal and Child Health Surveillance of China, Sichuan University

\section{Research Article}

Keywords: Congenital heart diseases, UGT1A1, Polycyclic aromatic hydrocarbons, 1-hydroxypyreneglucuronide, GMDR

Posted Date: May 6th, 2021

DOl: https://doi.org/10.21203/rs.3.rs-479397/v1

License: (9) This work is licensed under a Creative Commons Attribution 4.0 International License. Read Full License 


\section{Abstract}

Background: Previous studies have focused on the effect of polycyclic aromatic hydrocarbons (PAHs) exposure on the risk of congenital heart diseases (CHDs), but generated inconsistent conclusions. Genetic susceptibility to PAHs metabolism may modify the exposure-risk relationship.

Methods: Maternal urinary biomarker of PAHs exposure was determined in 357 pregnant women with CHDs fetuses and 270 pregnant women with controls. Maternal genotyping was conducted in UGT1A1 (rs3755319, rs887829, rs4148323, rs6742078, rs6717546). Unconditional logistic regression was performed to determine the impacts of UGT1A1 polymorphisms on the risks of CHDs and its subtypes. Generalized multifactor dimensionality reduction (GMDR) was used to analyze the gene-gene and genePAHs exposure interactions.

Results: None of the selected UGT1A1 polymorphisms were independently associated with the risk of CHDs. The interaction between rs4148323 and PAHs exposure was observed to be associated with CHDs $(p<0.05)$. Pregnant women with high-levels PAHs exposure and carrying rs4148323 increased the risk of CHDs (GA-AA: aOR $=2.00,95 \% \mathrm{Cl}=1.06-3.79$ ). Moreover, the joint effect of rs 4148323 and PAHs exposure was found to be significantly associated with risks of septal defects, conotruncal heart defects and rightsided obstructive malformations.

Conclusions: Maternal genetic variation of UGT1A1 rs4148323 may modify the impact of prenatal PAHs exposure on CHDs risk. This finding needs to be further confirmed in a larger scale study.

\section{Introduction}

Polycyclic aromatic hydrocarbons (PAHs) are ubiquitous environmental contaminants that are derived mostly from the incomplete combustion of tobacco, coal and other organic substances[1]. Exposed to PAHs is widespread for human beings through multiple routes such as inhalation of cigarette smoke or polluted air, and ingestion of food containing PAHs[2]. Concern on fetal hazards including congenital defects and intrauterine growth restriction after maternal exposure to PAHs has been raised[3-5].

Congenital heart diseases (CHDs) are among the most common of all human congenital defects. Growing evidences have indicated that PAHs can increase the risk of CHDs. Animal studies have shown that prenatal exposure to PAHs increases the occurrence of $\mathrm{CHDs}[6,7]$. Some epidemiological studies have also found women with prenatal exposure to PAHs are more likely to have offspring with CHDs such as conotruncal heart defects and septal defects[8], but a population-based study has shown null association[9]. Thus, we hypothesize that genetic variations such as metabolic enzyme gene polymorphisms influencing PAHs may partly be accountable for the inconsistent conclusions.

Some metabolic enzyme genes such as cytochrome P450 1A1 (CYP1A1) and uridine diphosphoglucuronosyl transferase 1A1 (UGT1A1) have been reported to modulate the metabolism and activation of PAHs[10,11]. Human studies have also observed that CYP1A1 and UGT1A1 gene 
polymorphisms significantly affect the concentrations of urinary 1-hydroxypyrene (1-OHP) as a biomarker of polycyclic aromatic hydrocarbons (PAHs) exposure[12, 13]. Moreover, maternal genotypes such as CYP1B1 polymorphisms have been shown to modify the association between maternal exposure to PAHs and CHDs risk[14]. However, the implications of UGT1A1 genetic polymorphisms in modulating the impacts of prenatal of PAHs exposure on the risk of $\mathrm{CHDs}$ remain undiscovered.

Here we utilized an established case-control study examining the impact of maternal PAHs exposure on the risk of CHDs, where prenatal PAHs exposure was associated with increased risk of $\mathrm{CHDs}[14]$. This study aimed to explore whether maternal UGT1A1 (rs3755319, rs887829, rs4148323, rs6742078, rs6717546) polymorphisms modified the association of CHDs risk with PAHs exposure.

\section{Materials And Methods}

\section{Study participants and data collection}

All pregnant women were selected from an established case-control study examining the impacts of maternal PAHs exposures on the risk of CHDs[14]. In brief, cases were having fetuses diagnosed with CHDs (without any extracardiac abnormalities), while controls in the same hospital were having fetuses with no major congenital malformations. Pregnancies with multiple births and fetuses diagnosed with chromosomal aberrations and syndromic diseases were excluded. All CHDs cases were confirmed by echocardiography, cardiac catheterization, surgery, or autopsy. CHDs cases based on the anatomic lesions were also classified into six subgroups: septal defects, conotruncal heart defects, left-sided obstructive malformations, right-sided obstructive malformations, anomalous pulmonary venous return, and other heart abnormalities[15]. The well-trained investigators administered a face-to-face interview with each pregnant woman at enrollment. Information on demographic characteristics, living environment, lifestyle habits, maternal reproductive history, maternal illness and drug use history, maternal diet and nutrition were collected by the trained investigators.

\section{PAH exposure assessment}

Urine samples from each pregnant woman were collected and stored at $-70^{\circ} \mathrm{C}$ until analysis. The 1hydroxypyrene-glucuronide (1-OHPG) in urine is a sensitive exposure biomarker for low-level PAHs exposure[16]. Thus, urinary 1-OHPG concentration was determined using liquid chromatograph system/tandem mass spectrometer mode as described elsewhere[14]. We used urine creatinine (cr) contents to adjust the concentration of 1-OHPG[17]. Moreover, the level of PAHs exposure was categorized into two groups (high and low exposure groups) according to the established the optimal cutoff value of $1-\mathrm{OHPG}(0.03 \mu \mathrm{g} / \mathrm{g} \mathrm{Cr})$ in our previous study[14].

\section{Polymorphism selection and genotyping}


Blood samples from each pregnant woman were collected and stored at $-70^{\circ} \mathrm{C}$. Genomic DNA was extracted using a QIAamp ${ }^{R}$ DNA Blood Mini Kit (Qiagen, Cat. No. 51106, Germany). DNA samples were then stored at $-80^{\circ} \mathrm{C}$ before further analysis. In our analysis, selection of single nucleotide polymorphisms (SNPS) in the UGT1A1 gene was in accordance with the following criteria: (1) previously reported to be significantly associated with some diseases or PAHs metabolism [18-22]. (2) minor allele frequency (MAF) of at least 0.05 in Chinese Han population. Five SNPs (rs3755319, rs887829, rs4148323, rs6742078, rs6717546) were selected in the UGT1A1 gene.

SNP genotyping was further performed using an improved multiplex ligation detection reaction (iMLDR) technique (Genesky Biotechnologies Inc., Shanghai, China). For quality control, we randomly selected $10 \%$ of samples to monitor the reproducibility of the assays, and the concordance was $100 \%$. Five cases and one control failed to be genotyped for UGT1A1 rs6717546. More detailed information about the studied UGT1A1 polymorphisms is provided in Additional file Table S1.

\section{Statistical analysis}

Descriptive statistics for maternal and fetal characteristics of the study participants were conducted. Parametric and nonparametric methods were respectively used to test the statistical significance for differences in categorical or continuous variables. Deviation from Hardy-Weinberg equilibrium (HWE) expectation in controls was analyzed by chi-square test, and $p<0.05$ indicated a deviation from equilibrium. We first utilized unconditional logistic regression analysis to investigate the associations of maternal UGT1A1 polymorphisms with CHDs risk. The associations of UGT1A1 genotypes with CHDs risk were also stratified by specific CHD subtypes. For genotype comparisons, homozygous wildtype served as the reference group to which heterozygotes and variant homozygotes were compared. Then, testing for gene-gene and gene-PAHs exposure interactions associated with CHDs was performed using generalized multifactor dimensionality reduction (GMDR, version 0.7, University of Virginia, USA), a test that detects and characterizes non-linear interactions among discrete genetic and environmental attributes[23]. Meanwhile, the identified effects of gene-PAHs exposure on the risks of CHDs and its subtypes were further analyzed by logistic regression, in order to obtain the aOR and $95 \% \mathrm{Cl}$. All logistic regression analyses were adjusted for maternal age (years; continuous), gestational week (weeks; continuous), housing renovation, exposure to a factory or landfill ( $<1000$ meters), cooking at home ( $\geq 4$ times/week), parental smoking or environmental tobacco smoke (ETS) exposure, maternal alcohol consumption ( $\geq 1$ time(s)/week), and use of folic acid supplements. Two-sided $p<0.05$ was considered statistically significant. Statistical analyses were calculated using Stata version 14.2 (Stata Corp LP., College Station, United States of America).

\section{Results}

\section{Characteristics of participants}


A total of 627 pregnant women (357 with CHDs fetuses and 270 controls) were enrolled in our study. Table 1 summarizes maternal and fetal characteristics of the study participants. Data on the environmental characteristics of the cases and controls revealed no significant differences, with the exception of cooking at home and folic acid supplement. Meanwhile, urinary 1-OHPG concentrations were significantly higher in cases $(P<0.001)$. In fetuses with CHDs, septal defects were the most frequent malformations (65.83\%), followed by conotruncal heart defects $(44.82 \%)$ and right-sided obstructive malformations (31.93\%). 
Table 1

Maternal and fetal characteristics among the study subjects

\section{Characteristic}

Maternal characterisics

\begin{tabular}{|c|c|c|c|}
\hline Maternal age (yrs) ${ }^{a}$ & $29(26,32)$ & $28(25,31)$ & 0.010 \\
\hline Gestational week (week) a & $23(18,25)$ & $25(23,27)$ & $<0.001$ \\
\hline Housing renovation ${ }^{\mathrm{b}}, \mathrm{n}(\%)$ & $52(19.26)$ & 79 (22.13) & 0.381 \\
\hline Factory or landfill nearby ${ }^{\mathrm{b}}, \mathrm{n}(\%)$ & $37(13.70)$ & $56(15.69)$ & 0.489 \\
\hline Cooking at home ${ }^{\mathrm{b}}, \mathrm{n}(\%)$ & $122(45.19)$ & $203(56.86)$ & 0.004 \\
\hline Parental smoking or ETS exposure ${ }^{\mathrm{b}}, \mathrm{n}(\%)$ & $155(57.41)$ & $223(62.46)$ & 0.200 \\
\hline Maternal alcohol consumption ${ }^{\mathrm{b}}, \mathrm{n}(\%)$ & $8(2.96)$ & $6(1.68)$ & 0.282 \\
\hline Folic acid supplements, $\mathrm{n}(\%)$ & $240(88.89)$ & $297(83.19)$ & 0.044 \\
\hline Urinary 1-OHPG levels $(\mu \mathrm{g} / \mathrm{g} \mathrm{Cr})^{a}$ & $0.32(0.06,0.74)$ & $0.42(0.15,0.88)$ & 0.006 \\
\hline \multicolumn{4}{|l|}{ Fetal characterisics (CHDs Subgroups) } \\
\hline Septal defects & & $235(65.83)$ & \\
\hline Conotruncal heart defects, n (\%) & & $160(44.82)$ & \\
\hline Right-sided obstructive malformations, n (\%) & & $114(31.93)$ & \\
\hline Left-sided obstructive malformations, n (\%) & & $72(20.17)$ & \\
\hline Anomalous pulmonary venous return, $\mathrm{n}(\%)$ & & $64(17.93)$ & \\
\hline Other cardiac structural abnormalities, n (\%) & & $102(28.57)$ & \\
\hline
\end{tabular}

a : Distribution was skewness, then Wilcoxon rank sum Z test was used, and described as interquartile range P50 (P25, P75)

b : The exposure was defined from the 3 months before pregnancy to the first trimester

\section{Association of UGT1A1 polymorphisms with CHDs}

For all five polymorphisms (rs3755319, rs887829, rs4148323, rs6742078, rs6717546) in UGT1A1, the distribution of the genotypes conformed well to the Hardy-Weinberg equilibrium (see Additional file Table 
S2). No significant association of UGT1A1 polymorphism with CHDs was found (Table 2). The rs4148323 genotypes with at least one A allele showed an increased risk of right-sided obstructive malformations (see Additional file Table S3), however, it was of borderline significance ( $\mathrm{OOR}=1.58,95 \%$ $\mathrm{Cl}=0.95-2.63)$.

\section{Table 2}

Association between maternal UGT1A1 polymorphisms and the risk of CHDs

Genotype Controls(n(\%)) Cases(n(\%)) aOR $^{\text {a }}(95 \% \mathrm{Cl})$

\section{rs3755319}

$\begin{array}{llll}\text { AA } & 123(45.56) & 152(42.58) & \text { Ref } \\ \text { A/C-C/C } & 147(54.44) & 205(57.42) & 1.12(0.79,1.58)\end{array}$

rs887829

$\begin{array}{llll}\mathrm{CC} & 214(79.26) & 286(80.11) & \text { Ref } \\ \mathrm{C} / \mathrm{T}-\mathrm{T} / \mathrm{T} & 56(20.74) & 71(19.89) & 0.98(0.64,1.50)\end{array}$

\section{rs4148323}

$\begin{array}{llll}\text { GG } & 193(71.48) & 263(73.67) & \text { Ref } \\ \text { G/A-A/A } & 77(28.52) & 94(26.33) & 1.07(0.73,1.57)\end{array}$

rs6742078

$\begin{array}{llll}\text { GG } & 212(78.52) & 284(79.55) & \text { Ref } \\ \text { G/T-T/T } & 58(21.48) & 73(20.45) & 0.94(0.61,1.44)\end{array}$

\section{rs6717546}

$\begin{array}{llll}\text { GG } & 117(43.33) & 143(40.06) & \text { Ref } \\ \text { G/A-A/A } & 152(56.30) & 209(58.54) & 0.92(0.66,1.28)\end{array}$

aOR = adjusted odds ratio, $\mathrm{Cl}=$ confidence interval

a : Adjusted for maternal age, gestational week, housing renovation, factory or landfill nearby, cooking at home, parental smoking or ETS exposure, maternal alcohol consumption, folic acid supplements.

b : Five cases and one control failed to be genotyped for UGT1A1 rs6717546.

\section{Gene-Gene and Gene-Environment Interaction}

We used GMDR to screen for the best interaction combination among five polymorphisms of the UGT1A1 gene (Table 3). A meaningful model involving rs4148323 and PAHs exposure was observed $(p<0.05)$. Overall, the cross-validation consistency of this model was 10/10, and the testing accuracy was 0.5698 
$(P<0.05)$. It indicated that there was a potential interaction between rs4148323 and PAHs exposure influencing CHDs risk. Although UGT1A1 rs4148323 was not associated with CHDs, pregnant women carrying A allele of rs 4148323 had a significantly increased risk of CHDs in the high-levels PAHs exposure $(\mathrm{aOR}=2.00,95 \% \mathrm{Cl}=1.06-3.79)$ (Table 4). Moreover, women with high-levels PAHs exposure and carrying rs 4148323 increased risks of septal defects, conotruncal heart defects and right-sided obstructive malformations (see Additional file Table S4).

Table 3

Gene-gene and gene-PAHs exposure interaction models in CHDs obtained using the GMDR method

\begin{tabular}{|llccc|}
\hline Interaction models & TBA1 & TBA2 & CVC & P value \\
\hline Gene-gene interaction & & & & \\
\hline SNP1 SNP5 & 0.5377 & 0.4487 & $7 / 10$ & 0.99 \\
\hline SNP1 SNP3 SNP5 & 0.5480 & 0.4314 & $7 / 10$ & 1.00 \\
\hline SNP1 SNP3 SNP4 SNP5 & 0.5599 & 0.4260 & $10 / 10$ & 1.00 \\
\hline SNP1 SNP2 SNP3 SNP4 SNP5 & 0.5605 & 0.4197 & $10 / 10$ & 1.00 \\
\hline Gene-PAHs exposure interaction & & & & \\
\hline SNP3 PAHs & 0.5711 & 0.5698 & $10 / 10$ & 0.01 \\
\hline SNP3 SNP5 PAHs & 0.5753 & 0.5308 & $4 / 10$ & 0.05 \\
\hline SNP1 SNP3 SNP5 PAHs & 0.5944 & 0.5185 & $10 / 10$ & 0.38 \\
\hline SNP1 SNP3 SNP4 SNP5 PAHs & 0.6060 & 0.4940 & $10 / 10$ & 0.38 \\
\hline SNP1 SNP2 SNP3 SNP4 SNP5 PAHs & 0.6069 & 0.4908 & $10 / 10$ & 0.38 \\
\hline SNP1: rs3755319; SNP2: rs887829; SNP3: rs4148323; SNP4: rs6742078; SNP5: rs6717546. & \\
\hline $\begin{array}{l}\text { TBA1: training balanced accuracy; TBA2: testing balanced accuracy; CVC: cross-validation } \\
\text { consistency }\end{array}$ & & & \\
\hline
\end{tabular}


Table 4

The interaction between maternal PAHs exposure and UGT1A1 rs4148323 influencing CHDs based on additive model

\begin{tabular}{|lllll|}
\hline rs4148323 & PAHs exposure & $\begin{array}{l}\text { Controls } \\
(\mathbf{n}(\%))\end{array}$ & $\begin{array}{l}\text { Cases } \\
(\mathbf{n}(\%))\end{array}$ & $\begin{array}{l}\text { aOR } \\
(95 \% \mathrm{Cl})\end{array}$ \\
\hline G/G & low & $41(15.19)$ & $29(8.12)$ & Ref. \\
\hline G/A-A/A & high & $152(56.30)$ & $234(65.55)$ & $1.74(0.98,3.06)$ \\
\hline & high & $56(20.74)$ & $80(22.41)$ & $2.00(1.06,3.79)$ \\
\hline aOR = adjusted odds ratio, Cl = confidence interval & & \\
\hline $\begin{array}{l}\text { a }: \text { Adjusted for maternal age, gestational week, housing renovation, factory or landfill nearby, cooking } \\
\text { at home, parental smoking or ETS exposure, maternal alcohol consumption, folic acid supplements. }\end{array}$ \\
\hline
\end{tabular}

\section{Discussion}

Congenital heart diseases (CHDs) ranks the leading cause of birth defect-related mortality[24]. To date, the etiology of CHDs has not been completely understood. It is widely believed that most CHDs are caused by a complex combination of genetic and environmental factors[25]. Our study investigated an underlying role of UGT1A1 gene on a previously identified association between PAHs exposure and CHDs risk[14]. In the current study, we found the association of PAHs exposure with CHDs risk was modified by rs4148323 polymorphism in the UGT1A1 gene.

The uridine diphosphoglucuronosyltransferases (UGTs) belong to a superfamily of metabolizing enzymes participating in detoxifying endogenous and exogenous compounds such as steroid hormones, xenobiotics and drugs[26, 27]. Several polymorphisms in UGT1A1 gene can affect expression and activity of encoded enzymes[27, 28]. In recent years, several studies have reported the associations of UGT1A1 gene variations such as rs4148323 and rs887829 with diseases risks, including neonatal hyperbilirubinemia and cancer. Two meta-analysis studies have shown that rs 4148323 polymorphism is a risk factor of developing neonatal hyperbilirubinemia in the Asian population, but not in the Caucasian population [21, 29]. For rs887829 polymorphism, one case-control study showed it reduces the risk of neonatal hyperbilirubinemia[18], but no association was observed with risks of endometrial cancer and gallstone[19, 30].

So far, there is limited research evaluating the impact of other UGT1A1 polymorphisms (rs3755319, rs6742078 and rs6717546) on disease risk. No significant association with progression-free survival or overall survival was observed with rs3755319 polymorphism in irinotecan-treated colorectal cancer patients[31]. As for rs6742078, it reduces the risk of new-onset type 2 diabetes in a Dutch population[20], but increases gallstone risk in German and Indian populations[30, 32]. As for rs6717546, a retrospective 
case control study found it is likely a protective factor against neonatal hyperbilirubinemia[18]. However, none of UGT1A1 polymorphisms was detected to be associated with the risk of CHDs in our study. We speculated that the heterogeneity observed between various diseases may be related to different expression of UGT1A1 in diverse organ sites, and affected by gene-environmental interactions.

The interaction of gene-environment has been investigated to explore the etiology of various diseases in previous studies. A meta-analysis has shown that UGT1A1 rs4148323 polymorphism increased the risk of severe neutropenia in the low dose of irinotecan (GA + AA vs. GG: OR $=2.66,95 \% \mathrm{Cl}=1.10-6.45, \mathrm{P}=$ 0.03) [33]. Another study in Japan found the effect of rs4148323 polymorphism on neonatal hyperbilirubinemia is observed in neonates with $5 \%$ or greater maximal body weight loss, increasing with the degree of maximal body weight loss[34]. However, a population-based study failed to find the joint effect of UGT1A1 rs887829 and soy food intake on the risk of endometrial cancer[19]. In our study, we demonstrated that maternal UGT1A1 rs4148323 was associated with increased CHDs risk in the highlevels PAHs exposure. Thus, it can be speculated that the inconsistency of conclusions of the association between PAHs exposure and CHDs might partly be the role of rs4148323 in the UGT1A1 gene. Given the relatively limited sample size, our finding is needs to be further confirmed.

Our study had several strengths. The study was the first to evaluate the effect of maternal UGT1A1 genetic polymorphisms on the risks of CHDs and its subtypes. Secondly, we used 1-OHPG in urine as a quantitative biomarker for estimating prenatal exposure to PAHs. Third, we examined the interactions of gene-gene and gene-PAHs exposure in CHDs by using the method of GMDR. However, several limitations should be noticed. First, limited sample size and multiple comparisons reduced statistical power to evaluate the risk of CHDs with UGT1A1 genetic polymorphisms and their combination with PAHs exposure. Second, a single spot urine measurement cannot precisely estimate the mother's long-term exposure level. Thus, future studies are needed to collect multiple urine samples. Third, fetal genotypes were not considered, and future studies are needed to investigate the effects of maternal and fetal genotypes, and gene-exposure interaction on the risk of CHDs.

\section{Conclusion}

Our study indicates that maternal genetic variation of UGT1A1 rs4148323 modified the association between PAHs exposure and CHDs. A more comprehensive, larger scale study is needed to further detect other genetic polymorphisms and gene-exposure interactions with respect to CHDs risk.

\section{Abbreviations}

PAHs: Polycyclic aromatic hydrocarbons, CHDs: Congenital heart diseases, UGT1A1: Uridine diphosphoglucuronosyl transferase 1A1, 1-OHPG: 1-hydroxypyrene-glucuronide, SNPs: Single nucleotide polymorphisms, GMDR: Generalized multifactor dimensionality reduction, TBA1: Training balanced accuracy, TBA2: Testing balanced accuracy, CVC: Cross-validation consistency, HWE: Hardy-Weinberg equilibrium, aOR: adjusted odds ratio, Cl: Confidence interval 


\section{Declarations}

Acknowledgements

We are grateful to the obstetricians, paediatricians, pathologists, experimental technicians and other participants involved in the project for recruiting the case and control participants and collecting the data.

\section{Author contributions}

Ping Yu, Jun Zhu, Fangfang Luo and Yanna Zou conceived of the study, participated in its design. Zhen Liu, Ying Deng, and Xiaohong Li performed data analyses. Jing Tao and Nana Li drafted the manuscript. All authors approved and agreed to be responsible for all aspects of the work ensuring integrity and accuracy.

\section{Funding}

This study is funded by The National key Research and Development Program of China (ID:

2016YFC1000102), Scientific research projects of Health Commission of Sichuan Province (ID: 16PJ247) and the Program for Changjiang Scholars and Innovative Research Team in University (ID: IRT0935), Science and Technology Project of Sichuan Province (2018SZ0269).

\section{Availability of data and materials}

The datasets used and/or analyzed during the current study are available from the corresponding author on reasonable request.

\section{Ethics approval and consent to participate}

The study was conducted under the approval of the Ethics Committee of Sichuan University (No. 2010004) and followed the tenets of the Declaration of Helsinki. Informed consent was obtained from all participants.

\section{Consent for publication}

All authors have read and approved the content, and agree to submit it for consideration for publication in your journal.

\section{Competing interests}

The authors declare that they have no competing interests.

\section{References}

1. Zhang Y, Tao S. Global atmospheric emission inventory of polycyclic aromatic hydrocarbons (PAHs) for 2004. Atmospheric Environment 2009;43(4):812-19. doi: 10.1016/j.atmosenv.2008.10.050 
2. Suzuki K, Yoshinaga J. Inhalation and dietary exposure to polycyclic aromatic hydrocarbons and urinary 1-hydroxypyrene in non-smoking university students. Int Arch Occup Environ Health 2007;81(1):115-21. doi: 10.1007/s00420-007-0188-x [published Online First: 2007/03/31]

3. Patel J, Nembhard WN, Politis MD, et al. Maternal occupational exposure to polycyclic aromatic hydrocarbons and the risk of isolated congenital heart defects among offspring. Environ Res 2020;186:109550. doi: 10.1016/j.envres.2020.109550 [published Online First: 2020/04/27]

4. Lupo PJ, Langlois PH, Reefhuis J, et al. Maternal occupational exposure to polycyclic aromatic hydrocarbons: effects on gastroschisis among offspring in the National Birth Defects Prevention Study. Environ Health Perspect 2012;120(6):910-5. doi: 10.1289/ehp.1104305

5. Choi H, Rauh V, Garfinkel R, et al. Prenatal exposure to airborne polycyclic aromatic hydrocarbons and risk of intrauterine growth restriction. Environ Health Perspect 2008;116(5):658-65. doi: 10.1289/ehp.10958 [published Online First: 2008/05/13]

6. Farwell A, Nero V, Croft M, et al. Modified Japanese medaka embryo-larval bioassay for rapid determination of developmental abnormalities. Arch Environ Contam Toxicol 2006;51(4):600-7. doi: 10.1007/s00244-005-0319-x [published Online First: 2006/09/30]

7. Incardona JP, Collier TK, Scholz NL. Defects in cardiac function precede morphological abnormalities in fish embryos exposed to polycyclic aromatic hydrocarbons. Toxicol Appl Pharmacol 2004;196(2):191-205. doi: 10.1016/j.taap.2003.11.026 [published Online First: 2004/04/15]

8. Patel J, Nembhard WN, Politis MD, et al. Maternal occupational exposure to polycyclic aromatic hydrocarbons and the risk of isolated congenital heart defects among offspring. Environmental Research 2020;186 doi: 10.1016/j.envres.2020.109550

9. Lupo PJ, Symanski E, Langlois PH, et al. Maternal occupational exposure to polycyclic aromatic hydrocarbons and congenital heart defects among offspring in the national birth defects prevention study. Birth Defects Res A Clin Mol Teratol 2012;94(11):875-81. doi: 10.1002/bdra.23071

10. Shimada T, Fujii-Kuriyama Y. Metabolic activation of polycyclic aromatic hydrocarbons to carcinogens by cytochromes P450 1A1 and 1B1. Cancer Sci 2004;95(1):1-6.

11. Yang $H$, Shi $Z$, Wang $X-X$, et al. Phenanthrene, but not its isomer anthracene, effectively activates both human and mouse nuclear receptor constitutive androstane receptor (CAR) and induces hepatotoxicity in mice. Toxicology and Applied Pharmacology 2019;378 doi: 10.1016/j.taap.2019.114618

12. Chen $B, H u$ Y, Jin $T$, et al. The influence of metabolic gene polymorphisms on urinary 1-hydroxypyrene concentrations in Chinese coke oven workers. Sci Total Environ 2007;381(1-3):38-46. doi: 10.1016/j.scitotenv.2007.02.021

13. Nerurkar P, Okinaka L, Aoki C, et al. CYP1A1, GSTM1, and GSTP1 genetic polymorphisms and urinary 1-hydroxypyrene excretion in non-occupationally exposed individuals. Cancer Epidemiol Biomarkers Prev 2000 9(10):1119-22.

14. Li N, Mu Y, Liu Z, et al. Assessment of interaction between maternal polycyclic aromatic hydrocarbons exposure and genetic polymorphisms on the risk of congenital heart diseases. Sci Rep 
2018;8(1):3075. doi: 10.1038/s41598-018-21380-3

15. Deng K, Liu Z, Lin Y, et al. Periconceptional paternal smoking and the risk of congenital heart defects: a case-control study. Birth Defects Res A Clin Mol Teratol 2013;97(4):210-6. doi: 10.1002/bdra.23128

16. Strickland P, D. K. Urinary 1-hydroxypyrene and other PAH metabolites as biomarkers of exposure to environmental PAH in air particulate matter. Toxicology Letters 1999;108(2-3):191-99.

17. Li M, Wang Q, Zhu J, et al. A simple analytical method of determining 1-hydroxypyrene glucuronide in human urine by isotope dilution with ultra performance liquid chromatography-tandem mass spectrometry. Anal Bioanal Chem 2017;409(6):1513-18. doi: 10.1007/s00216-016-0083-y

18. Zhou Y, Wang SN, Li H, et al. Association of UGT1A1 variants and hyperbilirubinemia in breast-fed full-term Chinese infants. PLoS One 2014;9(8):e104251. doi: 10.1371/journal.pone.0104251

19. Deming SL, Zheng W, Xu WH, et al. UGT1A1 genetic polymorphisms, endogenous estrogen exposure, soy food intake, and endometrial cancer risk. Cancer Epidemiol Biomarkers Prev 2008;17(3):563-70. doi: 10.1158/1055-9965.EPI-07-0752

20. Abbasi A, Deetman P, Corpeleijn E, et al. Bilirubin as a Potential Causal Factor in Type 2 Diabetes Risk: A Mendelian Randomization Study. Diabetes 2015 64(4):1459-69. doi: 10.2337/db140228/-/DC1

21. Long J, Zhang S, Fang X, et al. Association of neonatal hyperbilirubinemia with uridine diphosphateglucuronosyltransferase 1A1 gene polymorphisms: meta-analysis. Pediatr Int 2011;53(4):530-40. doi: 10.1111/j.1442-200X.2011.03337.x

22. Shin H, Kim J, Cheong H, et al. Functional Study of Haplotypes in UGT1A1 Promoter to Find a Novel Genetic Variant Leading to Reduced Gene Expression. Ther Drug Monit 2015 37(3):369-74.

23. Xu H, Xu L, Hou T, et al. GMDR: Versatile Software for Detecting Gene-Gene and Gene-Environ- ment Interactions Underlying Complex Traits. Curr Genomics 2016;17(5):396-402.

24. Mensah GA, Brown DW. An Overview Of Cardiovascular Disease Burden In The United States. Health Affairs 2007;26(1):38-48. doi: 10.1377/hlthaff.26.1.38

25. Krauss RS, Hong M. Gene-Environment Interactions and the Etiology of Birth Defects. Essays on Developmental Biology, Part A2016:569-80.

26. Riedmaier S, Klein K, Hofmann U, et al. UDP-glucuronosyltransferase (UGT) polymorphisms affect atorvastatin lactonization in vitro and in vivo. Clin Pharmacol Ther 2010;87(1):65-73. doi:

10.1038/clpt.2009.181 [published Online First: 2009/10/02]

27. Tukey R, Strassburg C. Human UDP-glucuronosyltransferases: metabolism, expression, and disease. Annu Rev Pharmacol Toxicol 2000;40 581-616.

28. Miners J, McKinnon R, Mackenzie P. Genetic polymorphisms of UDP-glucuronosyltransferases and their functional significance. Toxicology 2002(12):453-6.

29. Mehrad-Majd H, Haerian MS, Akhtari J, et al. Effects of Gly71Arg mutation in UGT1A1 gene on neonatal hyperbilirubinemia: a systematic review and meta-analysis. J Matern Fetal Neonatal Med 2019;32(10):1575-85. doi: 10.1080/14767058.2017.1410789 
30. Ravikanth VV, Rao GV, Govardhan B, et al. Polymorphisms in UGT1A1 Gene Predispose South Indians to Pigmentous Gallstones. J Clin Exp Hepatol 2016;6(3):216-23. doi: 10.1016/j.jceh.2016.08.004

31. Yu Q, Zhang T, Xie C, et al. UGT1A polymorphisms associated with worse outcome in colorectal cancer patients treated with irinotecan-based chemotherapy. Cancer Chemotherapy and Pharmacology 2018;82(1):87-98. doi: 10.1007/s00280-018-3595-7

32. Buch $S$, Schafmayer $C$, Volzke $H$, et al. Loci from a genome-wide analysis of bilirubin levels are associated with gallstone risk and composition. Gastroenterology 2010;139(6):1942-51 e2. doi: 10.1053/j.gastro.2010.09.003

33. Zhang $X$, Yin JF, Zhang J, et al. UGT1A1*6 polymorphisms are correlated with irinotecan-induced neutropenia: a systematic review and meta-analysis. Cancer Chemother Pharmacol 2017;80(1):13549. doi: $10.1007 /$ s00280-017-3344-3

34. Sato H, Uchida T, Toyota K, et al. Association of breast-fed neonatal hyperbilirubinemia with UGT1A1 polymorphisms: $211 \mathrm{G}>\mathrm{A}$ (G71R) mutation becomes a risk factor under inadequate feeding. J Hum Genet 2013;58(1):7-10. doi: 10.1038/jhg.2012.116

\section{Supplementary Files}

This is a list of supplementary files associated with this preprint. Click to download.

- Additionalfile20210429.docx 